\title{
The development of a robotic assembly cell during ESPRIT Project 5629
}

\author{
P.Guedes \\ Assistente, ISEP - Instituto Superior de Engenharia do Porto \\ Investigador, CCP - Centro de CIM do Porto \\ pguedes@ccp.up.pt
}

\begin{abstract}
The implementation of a robotic assembly cell and it's integration on a CIM environment creats a multitude of problems. The design for robotic assembly which is a complex interactive task between design, $\mathrm{CAD}$ and robot simulation and off-line programming groups, the development of flexible mechanical tools for the assembly robot, and the implementation of the assembly cell controller for monotoring and control of the assembly process in the CIM factory, are some of the difficulties to be solved. In this paper we present a description of the solution implemented in CCP - Centro de CIM do Porto, during the ESPRIT 5629 project, project approved in October 1995.
\end{abstract}

\section{Keywords}

CIM, Assembly Cells, Robotics, Machine Vision

\section{INTRODUCTION}

The development of a robotic assembly cell in a CIM perspective is not an easy task. We have to implement a flexible system that allows the quick changing of the cell assembly process by just changing the cell parameters, with the minimum set-up phase, which should be done almost in an automatic way.

The design for robotic assembly is an important topic that is directly connected with assembly cell development. A good representation of the assembly sequence is essential for this process. In order to define assembly plans, a method for gathering all the information concerning the assembly sequence of operations is essential. One can imagine the complexity of a large assembly. All the aspects concerning (i) the assembly characteristics, weight, length, and number of parts, (ii) part characteristics like stiffness, vulnerability to handling, shape, size, symmetry, quality, tolerances, weight, joining method (screwing, welding, snap, etc.), and (iii) 
the robot handling process involving composing direction, holding down during insertion, aligning, resistance to entrance, etc., should be considered.

We start this paper with a description of our assembly cell and then we present a small description of the integration of the assembly cell in the CCP factory. Since integration involves more then the working phase of the cell, we present a method for creating assembly sequences taking full advantages of our assembly system and CCP architecture. Some aspects of the cell implementation are also presented.

\section{ASSEMBLY CELL CHARACTERISATION}

The assembly cell robot is a SCARA with 4 DOF (Adept Three). This kind of robot imposes that almost all the assemblies are done in a vertical manner. The first part of the assembly is placed on a surface parallel to robot plane of movement and held by a dedicated device. This imposition makes the process of assembly planning easier. Another characteristic of the CCP's assembly cell is the use of artificial vision for helping the robot tasks. We have mounted a CCD camera in the robot connected to an industrial vision system (Cognex 4200EX).

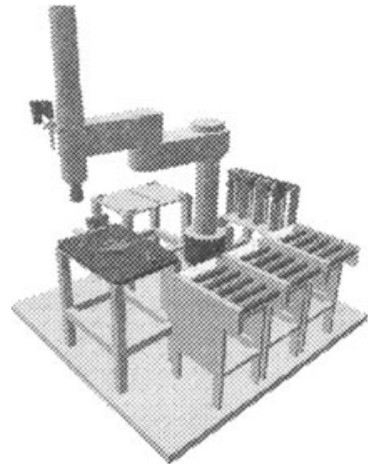

Figure 1 Assembly Cell

Attending to the robot structure and to the integration of the cell in the CCP complex, the cell was divided in different zones, based theirs'functionalities: operations table: this is where the assembly process takes place. This table is equipped with mechanical actuators for keeping the assembly stable; transfer tables: these transfer tables are the cell material feeding system.

The control of this system is not the responsibility of the assembly cell; gripper warehouse: warehouse based on a gripper change magazine with four positions; internal buffers : these tables are used for storage parts or subassemblies inside the cell perimeter, allowing more flexibility for the assembly process.

\section{ASSEMBLY CELL INTEGRATION}

The assembly cell is integrated in a larger system: "the CCP - CIM factory". Regarding the factory hierarchy (figure 2), we can see that the assembly cell just interfaces with the Shop Floor control. This interface is done by the assembly cell controller, which as two main 
objectives: first control the cell components, in our current case assembly robot and vision system, and second communicate with the Shop Floor Control, which controls the all factory.

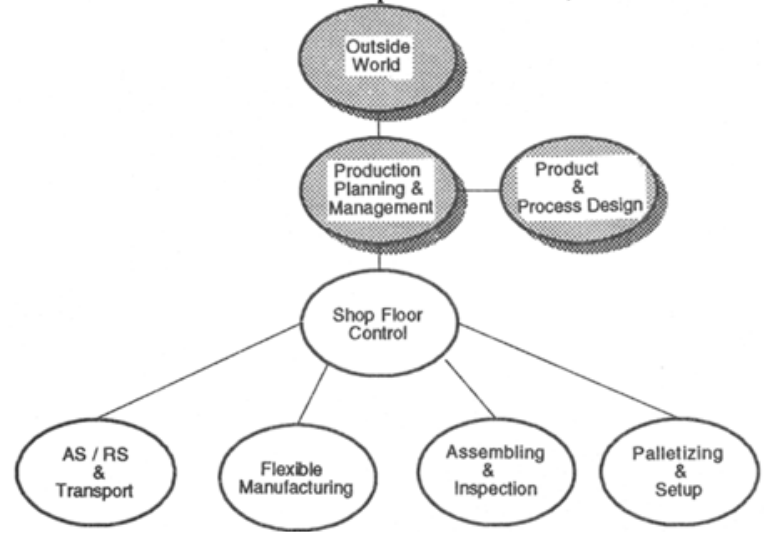

Figure 2 CCP factory hierarchy

The assembly cell control strategy is rather easy. The Shop Floor sends an assembly order to the cell. The cell controller processes the assembly order and looks for the necessary information for executing that assembly order. If it finds all the information, the order is accepted, if not the order is rejected. After obtaining the information, the cell controller decides which information should be sent to each of the components of the cell. When this setup phase is terminated, the cell controller is ready to start the assembly process. The cell controller is informed of all the operations executed by the robot controller, information that is processed and sent to the Shop Floor if necessary. The cell controller also sends the information the cell components needs during the assembly process regarding external events to the cell (e.g. the arrival of a container at a transfer table).

Unfortunately, we could not use new industrial communication standards, like MMS, in our cell as in all the others of our CIM platform. All the communications between cell controller and cell components done by RS232 serial line on a master/slave base.

\section{ASSEMBLY SEQUENCES DEVELOPMENT PROCEDURE}

\subsection{Robot trajectories}

An important topic for the work of the cell are robot trajectories (see table 1). The Adept controller calculates the trajectories between two points in two different ways: straight line between the two points or smooth trajectory between the two points. The programmer can only define if it is a straight line or if it is a normal trajectory. For the horizontal and oblique trajectories we need to verify the feasibility of the movement, which can be done in the off-line programming system for robots (IGRIP). The complex trajectories can be sampled into a 
group of passage points. The number of levels is defined by the precision we want for the trajectory.

Table 1 Robot trajectories

\begin{tabular}{ll}
\hline Trajectories & Description \\
\hline vertical & up and down with or without rotation. \\
horizontal & horizontal straight line or axe. \\
oblique & both vertical and vertical \\
complex & defined by a collection of points \\
\hline
\end{tabular}

Another important parameter to consider in trajectory planning is collisions. We have to consider collisions between the robot, the gripper and the handled part with the different components of the assembly cell. The existence of a robot off-line programming system simplifies this tasks. Here, with the real model of the cell, we can define and analyse the performance of different trajectories for the same assembly sequence.

\subsection{Degree of complexity in operations}

The complexity of the assembly operations involve the time needed for execution (includes gripper exchange time, vision calls time, etc.), the algorithms complexity (vision algorithms, trajectory planning, buffer space management, etc.) and the use of auxiliary devices (pneumatic screwdriver, pneumatic actuators, etc.). Many of these decisions are taken by a design team. They need a kind of heuristic that provides the choice of an assembly operation. Some of the topics that are important to that choice are presented in table 2 . The experience that we, in the robotics assembly group, has not allowed to decide for a good heuristic. Although, all this parameters have been taken in account in the implementation of the basic assembly operations described further in this document.

Table 2 Operations complexity

\begin{tabular}{ll}
\hline Operation topics & Description \\
\hline gripper change & yes, no \\
trajectory & vertical, horizontal, oblique , complex \\
precision & normal, high \\
vision routines & yes, no \\
vision complexity & normal, high \\
internal storage(cell) & yes, no \\
auxiliary devices & yes, no \\
what kind & pneumatic actuactors, screwdrivers, ... \\
\hline
\end{tabular}

\subsection{Degree of manipulability}

The degree of manipulability of part or subassembly, depends on its geometric shape, grasp points, and on the task to execute. Typically, small parts with simple geometry are easier to manipulate, although the integration of an assembly cell in a bigger system (production plant), forces us to consider a lot of factors that are exterior to the assembly cell. 
The shape of an object is essential for automatic assembly. The design for robotic assembly is a complex iterative process. The need of grip points well defined for complex parts, creates problems both to visual routines and handling operations. The definition of these points is done during the design phase. We can imagine that is not easy to create restrictions to handling points imposing physical changes to parts, and we cannot have a different robot gripper for every new part shape. Robotics assembly is much more flexible than automatic assembly, but we have also to define well for our system the area of application. In the CCP assembly cell, most of assemblies are done in a vertical manner. This reduces the manipulations and the grip points are easier to define. One can also conclude that with this kind of assembly, it is not easy to obtain a better degree of manipulability by doing alterations in the parts geometry.

\subsection{Subassemblies}

The division of an assembly in subassemblies is a complex task. This problem is more a design problem than an assembly problem. The parameters described in the previous subsections have to be taken in account in this task, but in our case we had also to consider several particularities that helps to the correct working of the production plant itself.

Two of the major limitations that we have in the CCP assembly cell are: (1) gripper change magazine with four positions, and (2) only three input/output transfer tables for an AGV. If one of the grippers is a pneumatic screwdriver, we have just three different grippers, each one for a reduced number of different part shapes. This is an important design topic. The other problem is more complex. If, for example, an assembly as more than three parts that arrives at the cell in different containers, the AGV has to provide the container exchange in one or more transfer tables. As the AGV transports materials to other cells, the assembly process duration can be random, which is not very good in a production plant. The subdivision of assemblies in small parts that are returned to the automatic warehouse is a solution for complex assemblies. Another solution, this one for assemblies comprising less that five grippers is kit production, i.e. , all the assembly parts arrive at the cell in the same container.

\subsection{Assembly sequences development procedure}

The development of an assembly sequence comprises synergy between all the design teams. The plan presented in figure 3 ) describes the information flux during the development process.

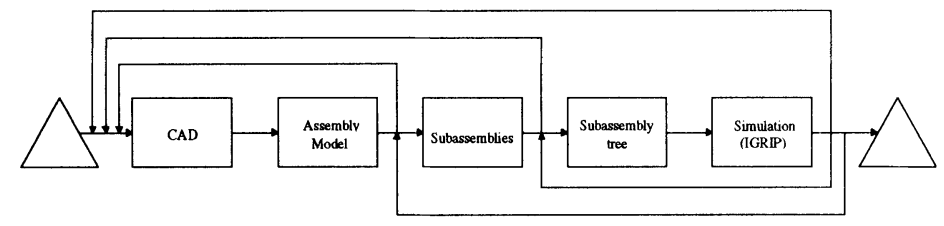

Figure 3 Assembly sequences development procedure

The first phase is the part and assembly design. Here the following rules have to be respected: privilege done to vertical assemblies, possible subassemblies with a small number of parts, minimisation of the development of news grippers or fixing devices, etc. The next phase, that can be or not realised by the CAD group, comprehends the definition of the assembly model. 
This models have the information concerning the parts (geometrical information, tolerances) and the complete assembly (which surfaces contact, joining methods, ...). From the study of this model one can decide if the piece need to be redesign. After we divide the assembly in subassemblies considering the manipulability of parts and the complexity of robot operations for that subassemblies. In this point one can decide to redesign some pieces of the assembly. Before the simulation phase we compile all the assembly sequence information in a subassembly tree: grippers, assembly trajectories, geometric models, actuator, etc. For the simulation in IGRIP, all the part geometric models are obtained from the CAD group. Here we intend to confirm if all the variables are suitable for the assembly process. Collision analysis is also performed.

This process is dynamic and based on the experience obtained from the different elements of the design team. The adoption of a philosophy based on simple rules and some heuristics, by one side allows the compilation of information in a quick and easy way, and by other side allows its expansion.

\section{IMPLEMENTATION TOPICS}

\subsection{Cell controller}

Based on the assembly control strategy, we have implemented the assembly cell controller in a modular perspective allowing easy expansion both on the assembly operations the robot can execute as on the components of the cell.

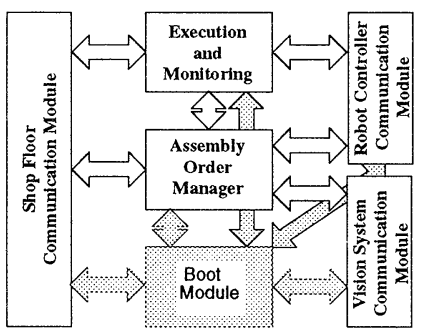

Figure 4 Cell controller architecture

Boot Module - This is the module for initialising all the other modules in the cell controller, verifying the state of the components of the cell, and starting the communication with the Shop Floor.

Assembly Order Manager - The main function of this module is the acceptance or rejection of assembly orders. It is responsible for processing the information inside the assembly order, passing it to the cell components and to the execution and monitoring module.

Execution and Monitoring - This module controls the assembly process. As control of execution and monitoring are connected, we have placed this functions in the same module. Other functionality of this module is managing the exceptions. 
Shop Floor, Robot Controller, Vision System Communication Modules - These modules manage the external communications of the cell controller. They format the messages to the different machines, initialise the communications between them, etc.

The communications between the different modules are based on a message passing and share memory. For adding new functions to the cell controller, we just have to create new messages and procedures for treat those messages. For adding new components to the cell, e.g. another robot, we have to incorporate a new Communication Module.

\subsection{Basic assembly operations}

In order to decrease the complexity of the assembly design and robot programming we have defined a group of basic robot assembly operations. An asembly program will be a sequence of these operations. Auxiliary functions, like gripper exchange, visual inspection tasks, opening and closing the operations table actuators, etc., are called inside of this basic operations. The designer does not have to worry about these problems, he just has to associate the necessary information to a part data structure, which is generated automatically by the robot off-line programming software.

HANDLING: Group of operations for moving parts inside the cell.

Pick-up: Operation used for picking up parts. The part can be on one of the cell tables or inside a container on the feeding system. Subtasks: verify the existence of the part; select the appropriate gripper; determine the position and orientation of the part; move to the pick-up place; activate the gripper;

Put-down: Operation used for putting down parts. Subtasks: verify if the place is free; move to the put-down place; activate the gripper;

STORAGE: The operations in this group are used for loading and unloading a container on a transfer table. After this operation the container is always dismissed from the cell.

Unload: Unload operation is used for picking up several parts from a container and putting them down on one of the internal buffers.

Load: Load operation is used for picking up several parts from one of the internal buffers and putting them down into container.

COMPOSING: Group of operations used directly in the assembly process.

Put-on: Operation used for putting parts on the operation table. This part is the first one of an assembly or subassembly. Subtasks: verify if the place is free; move to the put-on place; activate fixation system (mechanical actuators); activate the gripper;

Inserting: Operation used for total or partial insertion of a part in a subassembly. There is only one possible trajectory for the robot when this operation is executed. Subtasks: verify the insertion place; move to the insertion place;effectuate the insertion movement; activate the gripper;

Joining: Composing operation where one or more parts are joined together. Here we may have more than one degree of freedom for the robot trajectory. Subtasks: verify the joining place; move to the joining place; effectuate the joining movement; activate the gripper;

Screwing: Screwing is a typical connection operation. Using a pneumatic screwdriver with finder sleeve for picking up the screw, the robot is able to perform this operation. 
Subtasks: identify and pick-up screw; move to the insertion place; effectuate a controlled movement with the screwdriver;

Mounting o-ring: The use of o-rings is another way of connecting two cylindrical parts. This operation is a complex assembly tasks. Subtasks: identify a o-ring on the o-ring magazine; pick the o-ring in a vertical fashion; drop the o-ring in a controlled way over the operation table; pick the o-ring in horizontal position; move to insertion point, activate fixation; effectuate rotation around the insertion piece.

\subsection{Artificial vision}

The integration of artificial vision in the assembly process led us to the calibration of assembly cell and camera calibration and more precisely to hand-eye calibration. The CCD camera is placed in the second link of a SCARA type robot with 4 DOF. The flexibility needed in a robotic assembly cell, flexibility corresponding to the ability of changing the assembly process by a simple change of the sequence of basic assembly operations, associated with the multitude of interacting equipment with the assembly robot, induced us in choosing a generical 3D calibration for the robot-camera system.

The vision algorithms implemented to help robotic assembly tasks allows to calculate distances in robot's referential and to determinate the orientation of parts to be assembly. Part identification is also performed. One important characteristic of all the visual algorithms implemented is their adaptability to different light conditions, which does not impose any restriction to cell placement in a factory layout.

\section{CONCLUSIONS AND PERSPECTIVES}

We believe that the results of the CCP robotic cell implementation are a good start for further developments in the field. The adopted methodology has considered most of the implementation topics in the design and project phase and in the mechanical and software implementation, allowing a dynamic evolution of the cell based on the assembly needs and experience of the $\mathrm{CCP}$ robotic team.

In future, lots of improvements can be done in an assembly cell like this one. Including the vision system on the robot simulation, creating mechanisms for automatic error recover in conjugation with the cell controller, implementing automatic set-ups for the grippers warehouse by transporting the grippers in a container on the AGV, are some of the improvements we are thinking about.

\section{REFERENCES}

Guedes,P. (1994) Calibration of cameras mounted on robots.CCP Internal Report Guedes,P. (1995) Assembly sequences: a first approach to the problem. CCP Internal Report Guedes,P., Silva,M., Magalhães,P. and Baptista,J. (1995) The CCP assembly cell implementation.ESPRIT Project 5629: Presentation Day Proceedings, 117-133.

Silva,M. (1995) Simulation and Robot off-line programming of industrial robots. ESPRIT Project 5629: Presentation Day Proceedings, 169-189. 